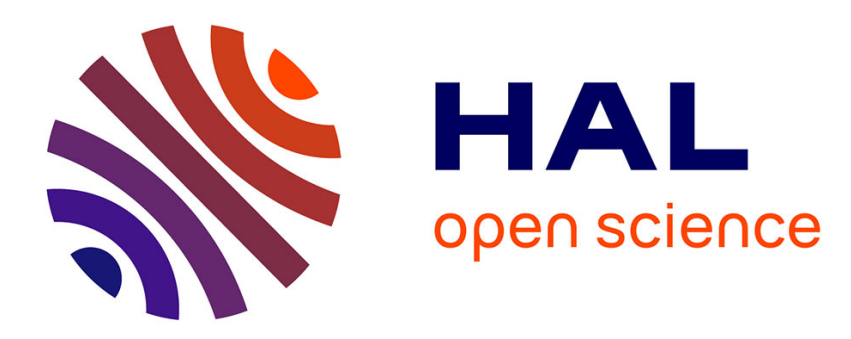

\title{
PAIR POTENTIALS AND THEIR LIMITATIONS FOR COMPUTER SIMULATIONS
}

\author{
R. Taylor
}

\section{To cite this version:}

R. Taylor. PAIR POTENTIALS AND THEIR LIMITATIONS FOR COMPUTER SIMULATIONS. Journal de Physique Colloques, 1985, 46 (C4), pp.C4-309-C4-320. 10.1051/jphyscol:1985434 . jpa00224684

\section{HAL Id: jpa-00224684 https://hal.science/jpa-00224684}

Submitted on 1 Jan 1985

HAL is a multi-disciplinary open access archive for the deposit and dissemination of scientific research documents, whether they are published or not. The documents may come from teaching and research institutions in France or abroad, or from public or private research centers.
L'archive ouverte pluridisciplinaire HAL, est destinée au dépôt et à la diffusion de documents scientifiques de niveau recherche, publiés ou non, émanant des établissements d'enseignement et de recherche français ou étrangers, des laboratoires publics ou privés. 


\title{
PAIR POTENTIALS AND THEIR LIMITATIONS FOR COMPUTER SIMULATIONS
}

\author{
R. Taylor
}

Division of Microstmatural Sciences, National Research Council of Canada, Ottowa, Ontario K1A OR6, Canada

\begin{abstract}
Résumé - La plupart des simulations sur orđinateur des structures et propriétés des défauts dans les cristaux utilisent des potentiels de paire. Notamment pour les simulations de joints de grain. La notion de potentiel de paire n'est pas difficile à justifier pour de nombreux systèmes mals pour d'autres, comme les métaux de transition en particulier, on peut légitimement mettre en doute sa validité. Cet article prèsente une revue de la notion de potentiel de paire, on discute de sa validité et de ses limites; en insistant particulièrement sur le cas des métaux.
\end{abstract}

Abstract - Most computer simulations of the structure and properties of defects in crystalline materials involve the use of pair potentials. This is particularly true of grain boundary simulations. In many systems the concept of a pair potential is not difficult to justify but in others, particularly transition metals, its validity can be serfously questioned. In this paper the pair potential concept is reviewed and its validity and limitations are discussed. The main emphasis throughout is on metals.

\section{I - INTRODUCTION}

A detailed theoretical understanding of the nature of defects and disorder in condensed matter very frequently entails an atomistic computer simulation of the materlal in question. This is because one is frequently interested in the structure and motions of particles near a defect site, or in a disordered material, in addition to the long-range strain fields which can usually be described by elasticity theory. No atomistic computer simulation can be performed without a suitable potential function which can be used to generate the total energy of the system for any possible configuration of particles relevant to the problem under consideration. Since such simulations generally are very time-consuming it is desirable to use a relatively simple algorithm to describe the energy of the system. The simplest possible procedure is to assume that all particles interact with each other via a pair potential which depends only on the relative separation of the particles. The energy is then constructed by summing over all pairs in the system. During the last two decades large numbers of pair potentials have appeared in the literature, many of which can be found in recent tabulations for ionics $/ 1 /$ and metals $/ 2 /$ and in the review by Aziz for rare gases $/ 3 /$.

The pair potential description of lonic systems, metals and rare gases is a very widely used approximation. Implicitly workers are assuming that $\mathrm{N}$-body forces $(\mathrm{N}>3)$ are either insignificant or can be averaged into an effective pair potentfal. In this paper I shall discuss the validity and limitations of this approximation when used for simulation of properties in the bulk material. Most of the discussion will be focussed on metals although there will be some comments on fonics and rare gases. I shall not discuss covalently bonded systems because the very nature of the bonding leads one to conclude that a pair potentlal description would he inademate. 
In the following two sections I shall review briefly the theory of interatomic pair potentials in simple metals and alloys and also discuss some limitations and characteristics of these potentials. In section 4 I shall discuss the validity of the pair potential concept in the much more complex transition metals. In section 5 the fitting of pair potentials to experimental data will be discussed. Finally in section 6 I shall summarlse the conclusions for metals and also discuss briefly the situation for lonics and rare gases. In view of the fact that this paper is being presented at a conference on internal interfaces particular attention will be paid to the question of the validity of pair potentlals for grain boundary simulations.

II - THEORY OF PAIR POTENTIALS IN SIMPLE METALS

Broadly speaking one can divide metals into two classes, simple metals and non-simple metals. Simple metals are the nearly-free-electron (nfe) metals. The ions consist of a nucleus surrounded by a tightly bound core of closed shells of electrons which do not overlap appreciably with those of neighbouring ions. These ions are immersed in a sea of nfe conduction electrons. Typical of such metals are the alkalis and AI. For most purposes one can also include in this group such metals as $\mathrm{Zn}$ or In which contain fairly tightly-bound d-electron shells forming narrow low-lying $d$-bands which have minimal influence on the conduction electrons.

Typical of the non-simple metals are the transition metals which have unfilled d-shells in the atoms which in the metal give rise to broad partially filled d-bands which have a profound influence on the properties of the metal. For this reason the concept of a pair potential in these systems is somewhat questionable and requires separate discussion.

An Intermediate group of metals consists of the noble metals, $\mathrm{Cu}, \mathrm{Ag}$ and $\mathrm{Au}$ and the alkaline earths, $\mathrm{Ca}, \mathrm{Sr}$ and $\mathrm{Ba}$. These systems have either completely filled or completely empty d-shells near the Fermi surface. The theory for them is rather more complex than for the simple metals $/ 4,5,6,7 /$ but the resulting potentials have the same basic characteristics as those of the simple metals $/ 4,8 /$ and hence for the purposes of discussion they will be grouped with them.

The theory of simple metal pair potentials can be summarised as follows. Starting with the schrodinger equation one mist consider three interactions, ( $I$ ) U $U_{I}^{b}(R)$, the bare Coulomb Interaction between ions, (11) $\mathrm{U}_{\mathrm{ee}}(\mathrm{x})$ the electron-electron interaction Including all exchange and correlation effects and (i1i) $U_{e I}(r, R)$, the electron-ion interaction. The symbols, $R$ and $r$, refer schematically to the ion and electron co-ordinates respectively. To solve the Schrodinger equation one invokes the adiabatic approximation which allows one to separate $/ 9 /$ the Hamiltonian into an effective ion Hamiltonian given by

$$
H_{I}=T_{I}+U_{I I}^{b}(R)+E(e, R)
$$

and an electron Hamiltonian

$$
\mathrm{H}_{\mathrm{e}}=\mathrm{T}_{\mathrm{e}}+\mathrm{U}_{\mathrm{ee}}(\mathrm{r})+\mathrm{U}_{\mathrm{eI}}(\mathrm{r}, \mathrm{R})
$$

where $T_{I}$ and $T_{e}$ are the fon and electron klnetic energles. $E(e, R)$ is the energy eigenvalue of $\mathrm{H}_{\mathrm{e}}^{\mathrm{e}}$ for a given fonic configuration and

$$
U_{I}^{(t o t)}(R)=U_{I I}^{b}(R)+E(e, R)
$$

represents an effective total potential energy for the ions. Hence the problem reduces to one of solving for the electronic energy, $E(e, R)$. In simple metals this is achieved by replacing $U_{e I}$ by a sum of pseudopotentials located on each ion site. In a formal sense these pseudopotentials should be constructed to give the same scattering phase shifts as the full electron-ion potentials that they are replacing. 
This can be done rigorously /10,11/ using the variable phase approach to scattering theory $/ 12 \%$. The pseudopotentials are then assumed to be sufficiently weak to be regarded as perturbations. Calculating $E(e, R)$ to second order in perturbation theory and inserting the result in (1) one obtains the well-known expression

$$
U_{I}^{\text {tot }}=U_{0}(p)+\frac{1}{2} \sum_{\ell, \ell^{\prime}}^{\prime} V_{I I}\left(\underset{\sim}{\mid R_{\ell}} \underset{\sim}{-R_{\ell}}, I, \rho\right),
$$

where the pair potential,

$$
V_{I I}(R, \rho)=\frac{(Z e)^{2}}{R}\left\{1-\frac{2}{\pi} \int_{0}^{\infty} F(q, \rho) \operatorname{sinqR} \frac{d q}{q}\right\} .
$$

$Z$ is the ionic valence, e the electronic charge and $F(q, \rho)$ the energy-wavenumber characteristic. Note the explicit dependence of the pair potential on the electron density, $\rho$. Also note the presence of the function $U_{0}(p)$ which is a potential independent of the structure and dependent only on the density. It is this potential which gives rise to the Cauchy pressure, proportional to $c_{12}-c_{44}$ in cubic systems, which holds the system in equilibrium.

For a local pseudopotential $F(q, \rho)$ can be written in the form

$$
F(q, \rho)=\frac{V_{p}^{2}(q)}{z^{2} 4 \pi e^{2} / q^{2}} \times(q, \rho),
$$

where $V_{p}(q)$ is the Fourier transform of the pseudopotential and $\chi(q, p)$ the susceptibility of the electron gas, given by

$$
\chi(q, p)=\frac{\Pi(q, p)}{\varepsilon(q, p)} ; \varepsilon(q, p)=1+\frac{4 \pi e^{2}}{q^{2}} \Pi(q, p) .
$$

$\varepsilon(q, \rho)$ is the electron gas dielectric function and $\Pi(q, \rho)$ the polarizability.

Strictly speaking we should be using a non-local pseudopotential. This would rise to a much more complicated expression for $F(q, \rho) / 13 /$. But for the purposes of this discussion it is not necessary to consider such details.

There is unfortunately an infinite set of pseudopotentials, all of which, when summed to all orders in perturbation theory, will give in principle the same answer 114/. From this set one must choose a pseudopotential which will ensure rapid convergence. One solution to this problem is to force convergence at the desired order of perturbation theory. Rasolt and Taylor $/ 15 /$ took this approach by requiring that the first order result for the charge density induced by an isolated ion in an infinite electron gas should agree with the fully converged nonlinear calculation. By this technique, pseudopotential parameters were determined for several simple metals $/ 13,15,16 /$. In essence this procedure sums all contributions to the pair potential from all orders of perturbation theory whilst retaining the mathematical simplicity of second order expressions. It also explicitly omits the effects of all three-body, four-body etc. potentials. These same ab initio pseudopotentials, suitably screened, have been used to calculate electron transport properties and self-diffusion energies of the alkalis as well as both harmonic and anharmonic phonon properties (for references see $/ 17,18 /$ ) with remarkably precise agreement with experiment being obtained. Similarly good results have also been obtained for Al $/ 19 /$. Hence one can conclude that for simple metals, a second order perturbation approach can produce a good unified treatment of a wide range of electronic and ionic properties. Therefore the pair potential concept, which is a consequence of second order perturbation theory, is well justified in these systems. 
Pair potentials for $\mathrm{II}$ and $\mathrm{Al}$ are illustrated in Figs. 1 and 2 for the low temperature dens $-y$ and for a density $20 \%$ lower in each case. Clearly evident are

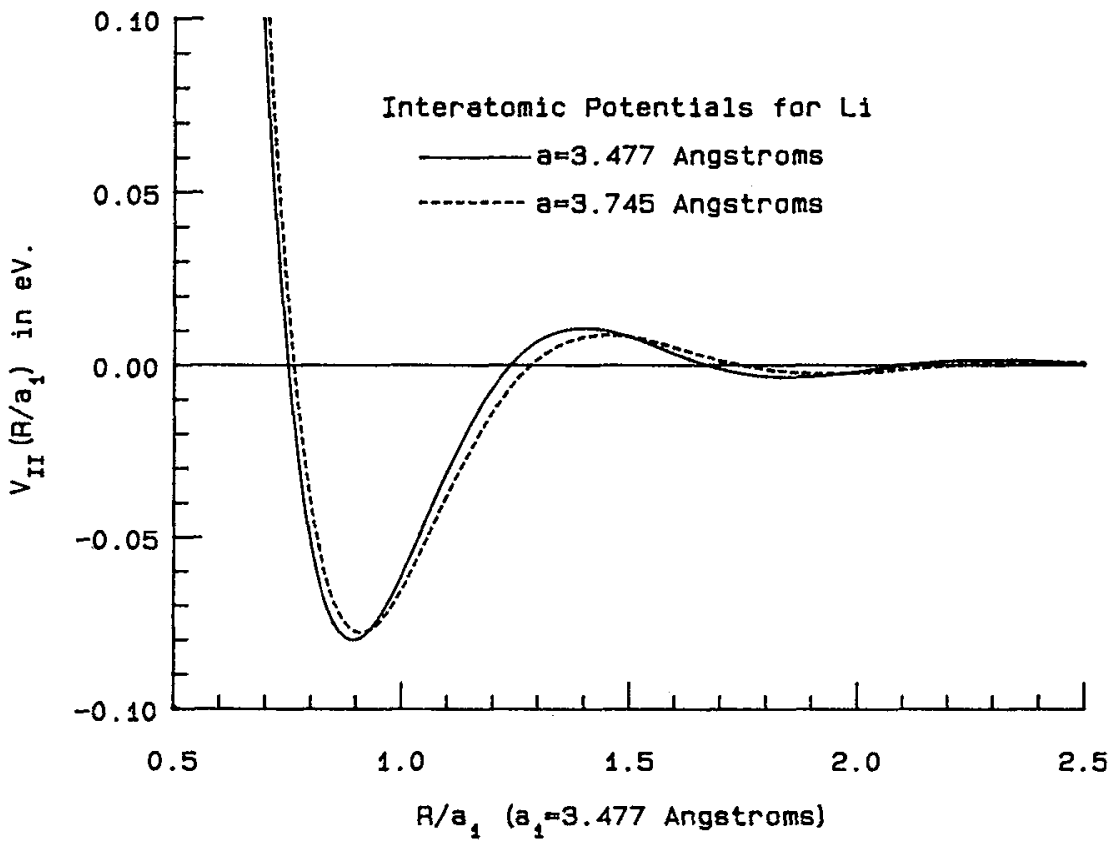

Fig. 1 - Interatomic pair potentials for $\mathrm{Li}$ calculated using the parameters of /16/ at two lattice parameters, one corresponding to low temperature and the other to a density $20 \%$ lower.

two important features, i.e. the density-dependence of the potentials and the presence of the well-known Friedel long-range osctllations. I.e., at large R,

$$
V_{I I}(R, \rho)=\frac{A_{3} \cos 2 k_{F} R}{\left(2 k_{F} R\right)^{3}}+\frac{A_{4} \sin 2 k_{F} R}{\left(2 k_{F} R\right)^{4}}+\cdots
$$

Both of these features can be very troublesome and require special attention.

The problem of density-dependence can be neatly circumvented for point defects by performing calculations at constant density. Using the equilibrium condition, it is easy to show that, at zero temperature, the vacancy formation energy at constant density is equal to the constant pressure result to order $N^{-1}$ where $N$ is the number of particles in the system /20/. In fact it follows from equation (2) that the vacancy formation energy is given by

$$
\Delta E_{v}=-\frac{1}{2} \sum_{\ell}^{\prime} V_{I I}\left(R_{\ell}, \rho\right)-\frac{1}{6} \sum_{\ell}^{\prime} R_{\ell} \frac{\partial V_{I I}\left(R_{\ell}, \rho\right)}{\partial R} .
$$

At finite temperature a correction must be added to calculate the formation enthalpy at constant pressure, $\Delta H_{p}$, due to the change in entropy when the system is allowed to expand after the vacancy is formed $/ 18 /$.

For more extended defects, such as grain boundaries, the problem of the density dependence of the potential could conceivably be more serious. In the neighbourhood 


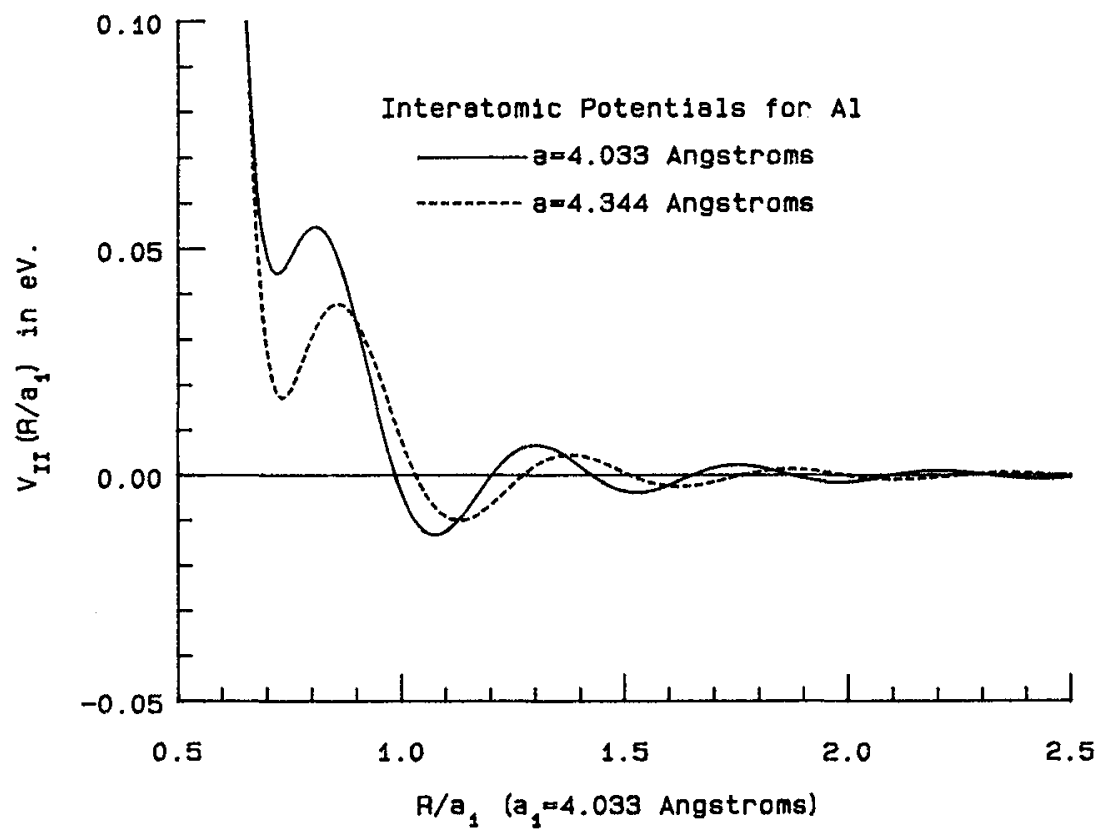

Fig. 2 - Interatomic pair potentials for Al calculated using the parameters of /16/ at two lattice parameters, one corresponding to low temperature and the other to a density $20 \%$ lower.

of a grain boundary the density of atoms, and of electrons, will be appreclably less than in the perfect crystal. Hence we are in a situation where the interactions across the grain boundary will differ from the bulk and also where the electron density is non-uniform. However the gradients in the charge density will be very much weaker than those encountered at a free surface. Within density functional theory, one can formally write the energy of a non-uniform electron system in terms of an expansion in powers of the gradients of the charge density /21/. Now it turns out that the linear response theory used to derive equations (2)-(5) is forma11y equivalent to the sum of an entire class of terms in the gradient expansion including the lowest order terms $/ 22 /$. Hence one can conclude that it is entirely valid to use interatomic potentials with a slowly varying charge density such as one might encounter at a grain boundary.

There still remains the question of what potential to use. The potentials in Figs. 1 and 2 were calculated at the zero temperature density and at a density $20 \%$ lower. Although there are significant changes in the potentials their functional form is unchanged. This is an important polnt in view of the calculations of Wolf for $A l$ and the noble metals $/ 23 /$ and of Budai et al. $/ 24 /$ for Au. These authors calculated grain boundary structures using many different pair potentials and found that the structures seemed to be largely independent of the fine details of the potential. Only if the functional form were profoundly different was the structure altered $/ 23 /$. Hence one can conclude that if one ignores the density dependence of the potential, the calculated grain boundary structure will not contain significant errors. The absolute energy, of course, will certainly be wrong. A possible remedy might be to calculate the average nearest neighbour distance to the fon under consideration and use the corresponding density to calculate the appropriate potential for that ion interacting with its neighbours. Such a procedure still remains to be tested and may be too tedious to be worthwhile. 
When using simple metal pair potentials one must treat the long range oscillations (6) carefully particularly when evaluating expressions such as (7) where the virial term behaves asymptotica11y as $\sin 2 \mathrm{k}_{\mathrm{F}} \mathrm{R} /\left(2 \mathrm{k}_{\mathrm{F}} \mathrm{R}\right)^{2}$. Arbitrary truncation of the potential can lead to very serfous errors. One procedure is to damp the oscillations /25/ to ensure suitably rapid convergence of potential sums. Another is to perform sums over the perfect system and defect system simultaneously and taking differences $/ 26 /$. The differences often converge more rapid1y than the individual sums.

Before closing this section it should be pointed out that there are situations where pair potentials are inadequate and three body forces play an important role. One example is the vacancy formation energy of a high electron density system such as Al where the potentlals near the vacancy site depend on whether or not the site is occupied /19/. Nevertheless vacancy migration in such a system is adequately described by unmodified pair potentials $/ 19,27 /$. In this context it is worth commenting that Solt $/ 28 /$ has provided a formulation beyond pair potentials that gives a good description of vacancy formation in Al. Conceivably his procedure could be adapted to the energy of a grain boundary.

III - PAIR POTENTIALS IN SIMPLE METAL ALLOYS

The equation for the simple metal pair potential (3) can easily be generalised to a two-component system /11/. For two species of ton labelled $\alpha$ and $\beta$ the potential takes the form

$$
v_{I I}^{\alpha \beta}(R, \rho)=\frac{z^{\alpha} z^{\beta} e^{2}}{R}\left\{1-\frac{2}{\pi} \int_{0}^{\infty} F^{\alpha \beta}(q, \rho) \text { sinqR } \frac{d q}{q}\right\},
$$

where $z^{\alpha}$ and $z^{\beta}$ are the respective valences of lons, $\alpha$ and $\beta$, and

$$
F^{\alpha \beta}(q, p)=\frac{v_{p}^{\alpha}(q) v_{p}^{\beta}(q)}{z^{\alpha} z^{\beta} 4 \pi e^{2} / q^{2}} \chi(q, p),
$$

which is the generalisation of equation (4).

For homovalent binary alloys the potentials quite clearly have the same validity as for the pure constituents of the alloys. Note that the potentials will be concentration dependent through the density-dependence of the potentials.

An interesting example of a successful homovalent alloy calculation is provided by a Molecular Dynamics simulation of localised phonon modes in a Rb-K alloy /29/. The calculated phonon frequencies for all phonon branches agreed very well with experimental data. In the analysis of the calculated results it was found that the individual ions in the alloy tended to relax signiflcantly off the perfect body-centred-cubic lattice sites. In fact this relaxation was responsible for $50 \%$ of the splitting of the localised $K$ phonon branch from the bulk [100] longitudinal branch. Such an effect would not be picked up by a KRR-CPA calculation where, by necessity, it is assumed that the alloy constituents sit at perfect lattice sites. Consequently the pair potential approach to alloys is far more useful than some KKR-CPA proponents would have us believe $/ 30 /$.

Turning to non-homovalent alloys the situation vis-à-vis pair potentials is not so clear. This is because of the possibility of charge transfer effects. In this context the LiMg alloy system is an interesting one to consider. Both components are classic examples of simple metals, well-described by pseudopotential theory. Hence one would expect pair potentials to work very well for structural properties. Beauchamp et al. $/ 26 /$ obtained good agreement with LiMg experimental data for elastic constants and heats of mixing as functions of alloy concentration. However the calculated short-range order parameters did not agree with experiment and this 
disagreement was attributed to possible charge transfer in the form of an increased pile up of charge around the $\mathrm{Mg}$ ions. In a later calculation Hafner $/ 31 /$ came to similar conclusions. However, recently, Gilder and Vignesoult /32/ re-examined this question and pointed out that by premature truncation of the LiMg potentials Beauchamp et al. had introduced an error in their calculation of short-range parameters. Redoing the calculation Gilder and Vignesoult found good agreement with experiment, leading to the suggestion that charge transfer effects are not significant in LiMg.

Clearly more work needs to be done before one can issue the blanket statement that the properties of simple metal alloys are well described by pair potentials. However it is worth pointing out that authors, such as Hafner /33-35/, have enjoyed considerable success in describing the properties of binary alloys using a pair potential description.

\section{IV - PAIR POTENTIALS IN TRANSITION METALS}

Transition metals are distinguished from simple metals by the fact that the corresponding free atoms contain a partly filled shell of d-electrons. When they come together in the metal these d-electrons form broad d-bands above and below the Fermi level, which interact strongly with the bands arising from the sp-valence electrons.

In a simple metal the conduction electrons tend to pile up around the ions, thereby screening them, and to follow the fon motion adiabatically. It is this effect that allows a pair potential description. In a transition metal the d-electron configuration is dictated by the minimisation of the d-band energy. When an ion is displaced, d-band energy minimisation will not necessarily lead to a cloud of charge following the ion around. Nevertheless, the desire to perform computer simulations in transition metals has led people to assume the existence of pair potentials and to determine the parameters by fitting to experimental properties. The very successful pioneering work of Johnson /36,37/ has led to the creation of large numbers of transition metal pair potentials without very much consideration of the theoretical basis of the pair potential concept.

Recently several authors /38-41/ have investigated the theoretical basis of pair potentials in transition metals and have attempted to construct potentials from band theory. In particular MacDonald and Taylor (MT) /41/ have attempted to provide an answer to the question of whether there is any validity to the description of the energy of a transition metal by a sum of overlapping pair potentials. Their approach was to consider only the $d$-band bonding energy of a model $d-b a n d$ metal within the tight binding approximation. The idea was to focus attention on that part of the energy that was least likely to be describable by pair potentials. Within that model they were able to write the energy as the sum over lattice sites of an effective pair potential which was formally structure-dependent. They then evaluated this 'pair' potential at the perfect lattice site as a function of the number of $d$-electrons $\left(N_{d}\right)$ for several different structures. For a given $N_{d}$ a plot of the results as a function of distance from the origin produced a curve that was surprisingly smooth. Generally speaking, for $3 \leqslant \mathrm{~N}_{\mathrm{d}} \leqslant 7$ there was very little scatter in the points. However, for other values of $N_{d}$, the scatter was more pronounced. As a rule the $\mathrm{fcc}$ and hexagonal structures generated points giving rise to a smooth curve, whilst the bcc and simple cubic structures were more 1ikely to generate points lying off the curve. From these results it was possible to conclude that for small changes in co-ordination number the pair potential concept was well-justified for transition metals. However it would seem unwise to rely on pair potentials to describe phase diagrams.

On the basis of their results MT suggested the following functiona1 form for a transition metal potential. 


$$
V_{I I}(R)=\frac{A e^{-B R / a}}{R / a}-\frac{C f_{d}}{1+\left(R / R_{1}\right)^{10} f_{d}}\left(\frac{a}{R}\right)^{5}
$$

where $A, B, C$ and $R_{1}$ are adjustable parameters, a is the lattice constant of an ideal fcc lattice at the same density as the system considered, and

$$
\mathrm{f}_{\mathrm{d}}=\mathrm{N}_{\mathrm{d}}\left(10-\mathrm{N}_{\mathrm{d}}\right) /\left(1+\mathrm{N}_{\mathrm{d}} / 50\right)
$$

The first term in (10) represents the screened Coulomb repulsion between the tons whilst the second term is the d-band bonding energy. The functional form of the latter gave a reasonable fit to the curves calculated from MT's tight-binding model. Note that this potential is density dependent; B is inversely to a screening length, $C$ is proportional to the bandwidth and $\mathrm{a}^{3}$ is proportional to the volume.

Equations (10) and (11) define a potential which is somewhat shorter ranged than that of Wills and Harrison $/ 40 /$ and which is qualitatively slmilar to Johnson's potential for iron $/ 36,37 /$. However Duesbery $/ 42 /$ reports that, like the Johnson potential, it suffers from the problem of predicting unstable dislocation kinks in bcc materials. It appears that an additional oscillatory term would be necessary to overcome this problem. Such a term could be justified on the basis that it would be analogous to the long-range oscillations found in the simple metal potentials. In equation (10) the effect of the sp-conduction electrons is contained entirely in the first term, whereas in fact an additional oscillatory term reflecting the effect of the Fermi surface should probably be included.

Another approach to calculating a transition metal pair potential is to fit the potential to the total energy calculated via a band structure procedure. A first attempt at this was suggested by Carlsson et a1. /43/ who simply inverted the energy as a function of density by assuming density-independent pair potentials and no separate density-dependent term in the energy. This scheme was only capable of producing a representation of the cohesive energy and could give no information about structures. A more realistic approach has recently been proposed by Machlin and Loh 144/. The idea is to calculate the total energy for fcc, bcc and simple cubic structures. Then write the differences between, say, fcc and bcc energies as

$$
E_{f^{-}}-E_{b}=\frac{1}{2} \sum_{\ell \ell^{\prime}}^{f c c} V_{I I}\left(R_{\ell \ell^{\prime}}\right)-\frac{1}{2} \sum_{\ell \ell^{\prime}}^{b c c} V_{I I}\left(R_{\ell \ell^{\prime}}\right)
$$

and similarly for other structures. This is done for several densities. The results are then fit to the form,

$$
V_{I I}(R)=\frac{A_{1} e^{-B_{1} R}}{R}+A_{2} \cos (2 k R+u) \frac{e^{-B_{2} R}}{R},
$$

where

$\mathbf{u}=\mathrm{C}+\mathrm{DS}$,

$S$ is the Wigner-Seitz cell radius and $A_{1}, A_{2}, B_{1}, B_{2}, C, D, k$ are fitting parameters. Applying this procedure to $\mathrm{Al}$, they produced a potential close to the solid curve in FIg. 2, which is encouraging. It remains to be seen what results this procedure generates for transition metals.

At this point it should be mentioned that one of the arguments against a pair potential is contained in an analysis presented by Heine $/ 45 /$. Heine showed that the second moment of the d-band energy is proportional to the co-ordination number $\mathrm{n}$. Hence the energy could be expected to be roughly proportional to $\sqrt{n}$ instead of $n$ as would be predicted by a pair potential approximation. Recognising this, Finnis and Sinclair /46/ have suggested the following procedure. Write the total energy as 


$$
\mathrm{U}_{\text {tot }}=\mathrm{U}_{\mathrm{N}}+\mathrm{U}_{\mathrm{p}} \text {, }
$$

where the repulsive core interaction takes the form

$$
U_{p}=\frac{1}{2} \sum_{i j} v\left(R_{i j}\right)
$$

and the tight-binding band energy takes the form

$$
U_{N}=-A \sum_{i} \sqrt{\rho_{i}} ; \rho_{i}=\sum_{j} \phi\left(R_{i j}\right) .
$$

Approximating $V\left(R_{i j}\right)$ and $\phi\left(R_{i j}\right)$ by polynomials cut of $f$ at short range, Finnis and Sinclair showed that they could obtain very reasonable unrelaxed vacancy formation energies for the 3d-transition metals after fitting parameters to various experimental quantities. As an additional comment, this procedure gives stable dislocation kinks in bec structure /42/. Approaches such as this may prove to be more viable than pair potentials in transition metals.

\section{V - FITTING PAIR POTENTIALS TO EXPERIMENT}

Because of the lack of pair potentials in transition metals generated by fundamental theory, users have been forced to resort to fitting functional forms to experimental data. Unfortunately, as has already been pointed out $147 /$, different workers fitting to essentially the same data can produce quite different potentials. Also there is a tendency to assume that the potential is short-ranged with an attractive we11 at the near neighbour distances. This assumption is certainly incompatible with theory for Al (see Fig. 2) and failure to realise this can lead to some strange results $147 /$. Al is not the only metal for which theory predicts a non-attractive pair potential at the nearest neighbour distance. Similar potentials have been calculated for $\mathrm{Be} / 2 /$ and $\mathrm{Ag}$ and $\mathrm{Au} / 8 /$. Hence one has to conclude that it is most Important to pay careful attention to the physics of the system for which a potential is to be constructed $/ 47 /$, and wherever possible to be guided by theory.

Recently Dharma-wardana and Aers $/ 48 /$ have been able to show that it is possible to generate very precise potentlals by fitting to liquid structure factors $\mathrm{S}(\mathrm{k})$. In their procedure they assume a physically motivated functional form for the potential and then use a modified hypernetted chain theory to calculate $S(k)$ for a given set of parameters. They then vary the parameters and repeat the process, eventually generating a least squares fit to the data. They first tested the procedure on a model A1 system for which $\mathrm{S}(\mathrm{k})$ was calculated by Molecular Dynamics (MD). By fitting to the MD $S(k)$ they recovered, very precisely, the potential that was used to generate it. (This type of test is strongly recommended for any fitting procedure.) Finally they fit to the experimental data and obtained a potential very similar to those of Fig. 2 .

In principle this approach could be applied to any system and these authors have used it to study $\mathrm{Kr} / 49 \%$ They obtained not only the pair potential but also information about the three-body potential, which gives a small correction to the pair potential. Unfortunately experimental data for two-component systems are not sufficiently precise to render this a practical method for anything other than a one-component system. It should however prove to be very useful in the understanding of transition metal potentials and in shedding light on non-pairwise forces in these systems.

Another procedure that has been used recently to obtain pair potentials in metals is to derive them from phonon spectra $/ 50-54 /$. The phonon data can only yield the first and second derivatives of the potential at the neighbour distances. To go beyond that, a functional form has to be assumed and fit to the derivatives. Brosens et al. $/ 50 /$ chose a polynomial in $\mathrm{R}^{2}$ whereas other authors $/ 51-54 /$ used polynomials in $R$. Generally the potentials obtained show good qualitative 
agreement, at the nearest neighbour distance, with the predictions of pseudopotential theory for simple metals, including Al $/ 51 /$, but were unable to reproduce the long-range oscillations satisfactorily. For that reason it is difficult to have great confidence in the potentials calculated in this manner for transition metals. However if a more physically motivated functional form were used for a potential, such as (10) or (13), this procedure might generate some very interesting results. It would also be interesting to test the procedure, in a similar fashion to $/ 48 /$, by taking a calculated phonon spectrum and seeing if the correct potential could be recovered. It is surprising that none of references /50-54/ applied this test.

\section{VI - SUMMARY AND DISCUSSION}

To summarise the previous sections, I have briefly reviewed the theory of interatomic potentials in simple metals and their alloys. Important points that emerge are that (i) the pair potential approximation is well-justified in simple metals and their alloys, (ii) pair potentials are density-dependent and exhibit a long-range oscillatory behaviour, (iii) ab initio theory can be used to generate potentials which. when handled properly give very good agreement with a wide range of experimental properties (e.g. /19/), (iv) empirical fits to metallic systems should incorporate as much physics as possible if one wishes to have confidence in the results $147 /$. In transition metals the pair potential concept is more difficult to justify but it appears that it is valid to use them to calculate at least the structural properties of defects, e.g. grain boundaries, where there is not a large change in co-ordination number from the perfect lattice. In general one expects transition metal potentials to be density-dependent but to be much shorter ranged than for simple metals. A particularly unfortunate feature of metallic pair potentials is their density dependence. However one can avoid this problem for point defects by doing constant density calculations. For more extended defects, such as grain boundaries, where it may be very important to allow for the volume change in order to incorporate complete relaxation $/ 23 /$, it still seems 1ikely that use of a density-independent potential will give a reasonable structure. However for free surfaces, where there is a very sharp gradient in the charge density, a pair potential approach is hard to justify.

The reason for concentrating on pair potentials in metals is that, because of the itinerant nature of the electrons, a semi-classical description of the ions might seem to be rather dubious. In fact it seems to be quite we11-justified. Hence in other systems, such as rare gases and ionics, where the electrons are well localised, one should feel quite confident that a pair potential description can be widely used. This is certafnly true for rare gas systems where pair potentials have a sound theoretical basis $/ 3 /$ and appear to be well understood.

In ionics, pair potentials are invariably constructed empirically /1/, but using models and functional forms that are soundly based on theory. The same can hardly be said of many metaliic potentials that can be found in the literature. Ionic potentials do not suffer from the problem of density-dependence, nor do they exhibit long-range oscillations. Hence computer simulations in such systems are much more straightforward, at least as far as the potential is concerned.

In short, for most computer simulations of bulk defect properties of metals, ionics and rare gas systems the pair potential concept is wel1-justified. In general the validity of a particular potential is limited by the degree to which the creator of the potential was able to make use of the physics of the system considered. 


\section{REFERENCES}

[1] STONEHAM, A.M., Handbook of Interatomic Potentials I. Ionic Crystals, AERE Harewe11 Report R 9598 (1979).

[2] STONEHAM, A.M. and TAYLOR, R., Handbook of Interatomic Potentials II. Metals, AERE Harwe11 Report R 10205 (1981).

[3] AZIZ, R.A., Inert Gases, ed. M.L. Klein, Springer Series In Chemical Physics 34 (Springer-Verlag BerIIn Heidelberg 1984) 5 .

[4] MORIARTY, J.A., Phys. Rev. B 6, (1972) 1239.

[5] MORIARTY, J.A., Phys. Rev. B 10 , (1974) 3075.

[6] DAGENS, L., J. Phys. F: Metal Phys. 6 (1976) 1801.

[7] DAGENS, L., Phys. Stat. Sol.(b) 84 (1977) 311.

[8] LAM, N.Q., DAGENS, L. and DOAN, N.V., J. Phys. F: Metal Phys. 13, (1983) 2503.

[9] VOSKO, S.H., TAYLOR, R. and KEECH, G.H., Can. J. Phys. 43 (1965) 1187.

[10] PENDRY, J.B., J. Phys. C: Solid St. Phys. 4 (1971) 427.

[11] RASOLT, M. and TAYIOR, R., J. Phys. F: Metal Phys. 2 (1972) 270.

[12] CALOGERO, F., Variable Phase Approach to Potential Scattering, (Academic Press New York 1967).

[13] TAYLOR, R. and MACDONALD, A.H., J. Phys. F: Metal Phys. 10 (1980) 2387.

[14] AUSTIN, B.J., HEINE, V. and SHAM, L.J., Phys. Rev. $127(\overline{1962}) 276$.

[15] RASOLT, M. and TAYLOR, R., Phys. Rev. B 11 (1975) $2 \overline{717}$.

[16] DAGENS, L., RASOLT, M. and TAYLOR, R., Phys. Rev. B 11 (1975) 2726.

[17] TAYLOR, R., Can. J. Phys. 60 (1982). 725 .

[18] JACUCCI, G. and TAYLOR, R., J. Phys. F: Metal Phys. 9 (1979) 1489.

[19] JACUCCI, G., TAYLOR, R., TENENBAUM, A. and VAN DOAN, N., J. Phys. F: Metal Phys. 11 (1981) 793.

[20] CHANG, R. and FALICOV, L.M., J. Phys. Chem. Solids 32 (1971) 465.

[21] HOHENBERG, P. and KOHN, W., Phys. Rev. 136 (1964) B $\overline{864}$.

[22] GELDART, D.J.W., RASOLT, M. and TAYLOR, R., Solid State Comm. 10 (1972) 279.

[23] WOLF, D., Acta Metall. 32. (1984) 245; 1bid. 32 (1984) 735 .

[24] BUDAI, J., BRISTOWE, P.D. and SASS, S.L., Acta Meta11. 31 (1983) 699.

[25] DUESBERY, M.S., JACUCCI, G. and TAYLOR, R., J. Phys. F: Metal Phys. 9 (1979) 413.

[26] BEAUCHAMP, P., TAYLOR, R. and VITEK, V., J. Phys. F: Metal Phys. 4 (1975) 2017.

[27] DA FANO, A. and JACUCCI, G., Phys. Rev. Lett. 39 (1977) 950.

[28] SOLT, G., Helv. Phys. Acta 54 (1981) 570.

[29] JACUCCI, G., KLEIN, M.L. and TAYLOR, R., Phys. Rev. B 18 (1978) 3782.

[30] FAULKNER, J.S., Progress in Materials Science, 27 (1982) 1.

[31] HAFNER, J., J. Phys. F: Metal Phys. 6 (1976) 1243.

[32] GILDER, H.M. and VIGNESOULT, S., private communication.

[33] HAFNER, J., Phys. Rev. B 15 (1977) 617.

[34] HAFNER, J. and VON HEIMENDAHL, L., Phys. Rev. Lett. 42 (1979) 386.

[35] HAFNER, J., Phys. Rev. B 21 (1980) 406.

[36] JOHNSON, R.A., Phys. Rev. 134 (1964) A1329.

[37] JOHNSON, R.A., Phys. Rev. $\overline{145}$ (1966) 423.

[38] CARLSSON, A.E. and ASHCROFT, N.W., Phys. Rev. B 27 (1983) 2101.

[39] HARRISON, W.A. and WILLS, J.M., Phys. Rev. B 25 (1982) 5007.

[40] WILLS, J.M. and HARRISON, W.A., Phys. Rev. B $\overline{28}$ (1983) 4363.

[41] MACDONALD, A.H. and TAYLOR, R.,, Can. J. Phys. 62 (1984) 796.

[42] DUESBERY, M.S., private communication.

[43] CARLSSON, A.E., GELATT, C.D. and EHRENREICH, H., Phil. Mag. A 41 (1980) 241.

[44] MACHLIN, E. and LOH, B., Phys. Rev, B 29 (1984) 3719.

[45] HEINE, V., Solid State Phys. 35 (1980) 1 .

[46] FINNIS, M.W. and SINCLAIR, J.E., A.E.R.E. Harwe11 Report T.P. 1030 (1983) and to be published.

[47] TAYLOR, R., Interatomic Potentials and Crystalline Defects, ed. J.K. Lee (TMS AIME Warrendale, PA 1981) 71 .

[48] DHARMA-WARDANA, M.W.C. and AERS, G.C., Phys. Rev. B 28 (1983) 1701.

[49] AERS, G.C. and DHARMA-WARDANA, M.W.C., Phys. Rev. A 29 (1984) 2734.

[50] BROSENS, F., CORNELIS, J., WALLACE, D.C. and DEVREESE, J.T., Phys. Stat. Sol. (b) 81 (1977) 557 . 
[51] ESTERLING, D.M. and SWAROOP, A., Phys. Stat. Sol.(b) 96 (1979) 401.

[52] SWAROOP, A. and ESTERLING, D.M., Phys. Stat. Sol.(b) 96 (1979) 691.

[53] VAN HEUGTEN, W.F.W.M., Phys. Stat. Sol. (b) 82 (1977) $\overline{501}$.

[54] VAN HEUGTEN, W.F.W.M. and DE HOSSON, J.TH.M., Phys. Stat. Sol.(b) 90 (1978) 225 .

\section{DISCUSSION}

R. Haydock: (i) One might think that the atomic sphere approximation would be bad for calculating three body forces. Have you tested its validity?

(ii) There have been recent measurements of three body forces of tungsten atoms on a tungsten surface by field ion microscopy. Could you comment on this?

R.Taylor: (i) You are referring to the model d-band metal calculation of MacDonald and myself in which I indicated that the atomic sphere approximation was used. This was just a means to implement our tight binding calculation. You are really asking if our model d-band system is a sufficiently good representation of the physics of a real d-band metal to draw conclusion about the validity of a pair potential description of the d-band bonding energy. The answer is yes, because it contains the qualitative features of real metal d-bands.

O.K. Andersen to question: (i) The atomic sphere approximation (including also the $s-$ and p-valence electrons) has been used to calculate structural energies (hcp, fcc, bcc, etc.) for a large number of elemental metals (simple-, transition-, rare earths and actinides) as a function of pressure. The results of these calculations by H.L. Skriver and others nearly always agree with the experimentally observed structures. This seems to indicate that the atomic sphere approximation contains (most of) the essential "physics".

R. Taylor: (ii) I neglected to mention in my talk that a simple pair potential description of the structures of metallic surfaces is not valid because of the sharp gradient in the electronic charge density. Hence, I would expect three-body forces to be present and there is no inconsistency with my comments on the validity of pair potentials in the bulk. 